\title{
GEOMETRIC ASPECTS OF GROUND AUGMENTATION OF SATELLITE NETWORKS FOR THE NEEDS OF DEFORMATION MONITORING
}

\author{
Elżbieta Protaziuk \\ Department of Engineering Surveying, Faculty of Geodesy and Cartography, \\ Warsaw University of Technology, Poland \\ e-mail: elka_protaziuk@poczta.onet.pl
}

\begin{abstract}
Satellite measurements become competitive in many tasks of engineering surveys, however, in many requiring applications possibilities to apply such solutions are still limited. The possibility to widely apply satellite technologies for displacements measurements is related with new challenges; the most important of them relate to increasing requirements concerning the accuracy, reliability and continuity of results of position determination. One of the solutions is a ground augmentation of satellite network, which intention is to improve precision of positioning, ensure comparable accuracy of coordinates and reduce precision fluctuations over time. The need for augmentation of GNSS is particularly significant in situations: where the visibility of satellites is poor because of terrain obstacles, when the determined position is not precise enough or a satellites constellation does not allow for reliable positioning. Ground based source/sources of satellite signal placed at a ground, called pseudosatellites, or pseudolites were intensively investigated during the last two decades and finally were developed into groundbased, time-synchronized transceivers, that can transmit and receive a proprietary positioning signal. The paper presents geometric aspects of the ground based augmentation of the satellite networks using various quality measures of positioning geometry, which depends on access to the constellation of satellites and the conditions of the observation environment. The issue of minimizing these measures is the key problem that allows to obtain the position with high accuracy. For this purpose, the use of an error ellipsoid is proposed and compared with an error ellipse. The paper also describes the results of preliminary accuracy analysis obtained at test area and a comparison of various measures of the quality of positioning geometry.
\end{abstract}

Keywords: deformation monitoring, quality measures of positioning geometry, ground based augmentation, pseudolite

\section{INTRODUCTION}

Issues related to determination of displacements and, in particular, structural deformation monitoring, are specific tasks in the area of geodetic surveys. Results of such surveys influence the safety of investigated structures and, in many cases, also the human life. Values of displacements determined as results of these surveys, must be characterized, first of all by: 
1. correctness - compliance with real variations of positions of observed points, within the limits of influence of random errors;

2. the minimum accuracy, justified by specific demands;

3. up-to-dateness - i.e. the possibly shortest time between the surveys and transfer of their results, (Lazzarini, 1977).

The above order is reasonable - the compliance between results and the reality is always the most important requirement, but, at the same time, its verification is the most difficult. For many tasks the accuracy may be of the secondary importance. Besides achieving the accuracy, contemporary trends of surveys and calculations of displacements aim at the reliability, which - in the case of measurements of displacements - is connected with ensuring the external, stable reference system. GPS as a reliable, versatile, usually available and relatively accurate positioning technology, able to operate anytime and anywhere across the globe seems to be competitive technology for this purpose.

The general tasks of geodesy, measurements of displacements are characterised by:

- higher, diversified and complicated requirements concerning accuracy. These requirements are mostly formed not by the accuracy of determination of point positions, but by the accuracy of determination of the structure conditions at the particular moment, referred to the initial moment. this relates both, to the geometric features of the structure, as well as to its location with respect to the surrounding structures (displacements and deformations). The accuracy of survey methods should be stable in time and in space within the entire process of the structure monitoring, it should be also adapted to the characteristics of the investigated structure;

- high requirements concerning the reliability - the necessity to effectively detect and eliminate gross errors from observations;

- specific environment of observations: challenging and variable (construction sites, tunnels, factory floors, mine workings); visibility limited by structures located within the area of engineering works, being the subject of these works and other structures (buildings, structures, vegetation); variable terrain morphology, slopes, steep banks, valleys directed to the south; disturbances of measurements, dust, vibrations, radio signal emission by electronic installation;

- the needs to perform periodical or continuous measurements;

- the need to automate processes of measurements and calculations.

The conventional surveys of angles and distances have the leading role in geodetic surveys of displacements. However, due to the high precision of phase, differential observations, the GPS technology, which has been applied in many tasks of geodesy for a long time, is also more and more often applied in tasks of engineering geodesy, including the measurements of displacements. In many cases, where the submillimetre accuracy is not required, satellite measurements become popular - in particular, in tasks related to settlement and slide of grounds. They are also often applied for monitoring deformations of various engineering structures, such as bridges, dams or buildings. The lack of visibility between measured points, weaker relations between the accuracy of measurements from the vector length allow for connection to reference points, which are located far from the structure without loosing the accuracy. It is possible to achieve almost continuous results, as well as to perform measurements in the case of limited visibility (e.g. fog, rainfalls, night). Due to these features satellite measurements are become competitive in many tasks for conventional measurements, however, in many applications (where the high precision is required, or within urban environment) possibilities to apply such solutions are still limited.

As it is known, availability, accuracy, reliability, integrity and continuity of positioning with the use of the GPS highly depend on the number and distribution of visible satellites. In 
practice, in many situations may occur that the GPS system is not able to ensure the sufficient accuracy and reliability of results because of its systemic limitations. In the case when obstacle obstruct the signal, implementation of satellite measurements becomes even impossible.

There are various methods for augmentation GPS system and they depend on the availability of GPS satellites constellation, the character of measurements and on the observation environment. One of such methods employs ground-based source/sources of positioning signal placed at a point whose coordinates are known. These devices being an additional source of signal with varied geometrical properties, could solve the problems deriving from the imperfections of the GPS system by strengthening the geometry positioning and increasing the number of signals available for measurement.

\section{GPS LIMITATIONS}

The basic limitations of the GPS measurements in displacements determination concern:

- the accuracy depending on the current positions of satellites, variable in time. Movements of the satellites cause their visibility changing in time or variable locations and the number of visible satellites influence the positioning accuracy. Therefore, depending on the currently visible satellites, the accuracy may also vary within a daytime;

- the decreased accuracy coordination between particular coordinates: the lower accuracy of vertical coordinate determination and lower accuracy of the north coordinate. Placing all sources of signals about $10-15^{\circ}$ above the horizon, with respect to the station, is not favourable for determination of vertical coordinate. As a result, the accuracy of the elevation coordinate is 2-3 times lower that the accuracy of horizontal coordinates. Within the areas of medium and high geographic latitude $\left(>45^{\circ}\right)$, where Poland is also located, due to the inclination angle of GPS satellites $\left(55^{\circ}\right)$ observations from the northern sky (between azimuths of $315^{\circ}$ and $45^{\circ}$ ) origin from satellites located close to the zenith, or, in majority of cases, from low satellites. In case of applying $10-15^{\circ}$ masks this makes observations from this direction impossible. Therefore, the observation hole is created, which is not favourable for the accuracy of determination of $\mathrm{N}$ coordinate (Meng et al. 2004). If, additionally, terrain obstacles would exist, which could limit the visibility of satellites from the southern direction, positioning could be difficult;

- limitations in the visibility of satellites by terrain obstacles. Terrain obstacles often occur within the area of activities related to engineering tasks - buildings, structures and constructions disturbing signals, do not allow to directly reach the receiver, limiting the number of visible satellites (often below the required number of four visible satellites). As a result, in difficult observation environment (first of all, in cities, deep open mines, areas close to engineering structures or areas covered by trees) the decreased number of visible satellites may result in lowered accuracy and reliability or it may be even insufficient for position determinations;

- basically, GLONASS, GALILEO systems have the same systemic limitations;

- besides difficulties related to geometry, other limitations also occur, such as, the increasing multipath issue, the issue related to locating points on the structure surface, disturbances of signals etc.

As it was already mentioned, issues related to the quality of geometry of the visible constellation of satellites, directly influence the possible accuracy and reliability of results. Application of methods of preliminary analysis of accuracy - the typical procedure used in engineering surveys - in the process of planning satellite measurements, allows detailed analysis of the quality of positioning geometry and identify its lacks. 


\section{QUALITY MEASURES OF POSITIONING GEOMETRY}

All systematic and random pseudoranging errors propagate into the solution by the geometry of the observation constellation, which is defined by distribution of satellites. Observational equations, expressed in the local coordinate system connected with the point of observations $(N, E, V)$ for $k$ observed satellites, create the following design matrix $A_{l o c}$ :

where:

$$
A_{l o c}=\left[\begin{array}{cccc}
\sin z_{i}^{1} \cos \alpha_{i}^{1} & \sin z_{i}^{1} \sin \alpha_{i}^{1} & \cos z_{i}^{1} & 1 \\
\sin z_{i}^{2} \cos \alpha_{i}^{2} & \sin z_{i}^{2} \sin \alpha_{i}^{2} & \cos z_{i}^{2} & 1 \\
\sin z_{i}^{3} \cos \alpha_{i}^{3} & \sin z_{i}^{3} \sin \alpha_{i}^{3} & \cos z_{i}^{3} & 1 \\
\cdots & \ldots & \ldots & \ldots \\
\sin z_{i}^{k} \cos \alpha_{i}^{k} & \sin z_{i}^{k} \sin \alpha_{i}^{k} & \cos z_{i}^{k} & 1
\end{array}\right]
$$

$z$ - zenith angle of the satellite,

$\alpha$ - the azimuth of the satellite in the local coordinate system.

The matrix of cofactors of the form (2) reveals the characteristic of errors propagation, which may be used to forecast the possible accuracy and planning the measurements. It changes in time together with the variations of satellite positions, limitation of their visibility and as a result of changing positions of the receiver.

$$
Q_{l o c}=\left(A_{l o c}{ }^{T} A_{l o c}\right)^{-1}=\left[\begin{array}{cccc}
Q_{N N} & q_{N E} & q_{N V} & q_{N T} \\
q_{N E} & Q_{E E} & q_{E V} & q_{E T} \\
q_{N V} & q_{E V} & Q_{V V} & q_{V T} \\
q_{N T} & q_{E T} & q_{V T} & Q_{T T}
\end{array}\right]
$$

Based on this matrix various quality measures may be calculated. An expected accuracy of determination of a point may be approximated by multiplication of the value of the corresponding measure by the standard deviation of the pseudorange between a satellite and receivers. Because elements of satellite orbits are known, their position with respect to the point of observations may be calculated for any moment. Therefore, it is possible to forecast quality measures related to the planned position and time of observations, as well as to forecast the possible accuracy of results. For long sessions the geometry may be considerably changed and its final influence on position determination will be averaged. On the other hand, in the case of short sessions or in the case of continuous monitoring the geometry of positioning for a particular epoch of observations may directly influence the accuracy of results.

Commonly, as factors of the quality of satellite constellation geometry are applied DOP (Dilution of Precision) values (Massat, Rudnick, 1990). DOP values may be calculated basing on the elements of the trace of cofactor matrix (2), Particular DOP values are determined as the root of the elements of the main diagonal:

- NDOP, EDOP, VDOP for determination of coordinates,

- TDOP - for determination of time,

- PDOP - for determination of spatial coordinates (3D),

- GDOP - for determination of spatial coordinates and time,

In typical geodetic surveys, it is assumed that if the PDOP value falls within the interval 1-3 conditions of observations are very good, they are satisfactory when this value equals to $4-5$, and when it reaches 6 , poor conditions of observations occur; when the PDOP value exceeds 6 , observations should not be performed. For the purpose of precise applications e.g. 
displacement monitoring it should be adopted first interval - very good conditions of observations, so therefore PDOP values should not exceed 3. DOPs has been widely used as an accuracy metric for navigation and tracking systems. The issue of optimization and reduction DOP coefficients is subject to a number of studies (Sharp et al. 2009, Ning et al. 2007, Morales et al. 2016). Although the DOP values are standard measures applied for determination of the quality of satellites geometry, it concerns only elements located in the main diagonal of the matrix (2), which are responsible for the accuracy of particular coordinates.

For precise applications, especially planning the measurements, more precise accuracy characteristic is recommended, which consider correlation between the coordinates, so also other elements, located outside the diagonal of cofactor matrix, i.e. error ellipse - typically used for describing accuracy.

The ellipse of errors shows distribution of accuracy characteristic in the horizontal plane, pointing to the direction of the lowest accuracy. The vertical accuracy is defined separately. That type of characteristics are typically used in traditional surveying methods, including the measurement of displacement. Horizontal and vertical displacements are usually determined from independent observations (tacheometry and precise leveling). In satellite measurements horizontal and vertical coordinates are determined together, using the same observation, and thus they are correlated.

Taking into account the correlation between all the coordinates requires the use of the error ellipsoid. It shows the spatial distribution of the accuracy characteristics.

To receive parameters of an error ellipse or ellipsoid, eigenvalues and eigenvectors of appropriate subset of the model parameters should be calculated, by taking relevant rows and columns of cofactor matrix (2)

- for horizontal error ellipse - $\mathrm{N}$ and $\mathrm{E}$ rows and columns - form (3)

$$
Q_{l o c N E}=\left[\begin{array}{ll}
Q_{N N} & q_{N E} \\
q_{N E} & Q_{E E}
\end{array}\right]
$$

The eigenvalues of matrix (3) are the squares of the semimajor and semiminor axes of the ellipse and the corresponding eigenvectors show the orientation of the error ellipse.

- for 3-dimensional error ellipsoid - N, E and V rows and columns - form (4):

$$
Q_{\text {locNEV }}=\left[\begin{array}{lll}
Q_{N N} & q_{N E} & q_{N V} \\
q_{N E} & Q_{E E} & q_{E V} \\
q_{N V} & q_{E V} & Q_{V V}
\end{array}\right]
$$

The eigenvectors define the principal axes of the ellipsoid and associated eigenvalues are the squares of the semi-axes. The eigenvector associated with the largest eigenvalue represents a slope of the major axis of an ellipsoid. The eigenvector of second largest eigenvalue represents a slope of the second axis of an ellipsoid and the eigenvector of smallest eigenvalue represents a slope of the minor axis of an ellipsoid. Correlation for parameter pairs are measures of the inclination of the error ellipsoid with respect to the parameter axes. A correlation approaching +1 means the projection is needle-like with its long principal axis having a positive slope, and a correlation approaching -1 means that the projection is needlelike with its long principal axis having a negative slope (Aster et al. 2012). The sum of the eigenvalues is always equal to the sum of the diagonal elements of the original matrix. The best situation is when the axes are almost equal, then the ellipse and ellipsoid approaching respectively the circle and the sphere.

Characteristics described above based on the matrix (1) refer to single point positioning. In order to determine the quality of the geometry of double differencing for pseudorange and 
carrier phase observations RDOP (relative dilution of precision) are applied - however, they do not have an explicit definition. Two definitions for RDOPs are given, one assuming fixed ambiguities and the other assuming floating ambiguities. Since basically the accuracy of differential positioning remains in close relation with the DOP values for each point (Rzepecka et al. 2005), DOPs are mostly applied for evaluation of the quality of geometry. The similar situation is in the case of relative error ellipses and error ellipsoids.

\section{ACCURACY PREANALYSIS FOR THE SIMULATED TEST NETWORK}

This chapter includes an exemplary analysis of available geometry of positioning and differences in various quality measures of geometry, in a particular observing condition. For needs of analysis a network of four points was located within the campus of the AGH (University of Science and Technology in Kraków). These points were evenly distributed within a rather open-sky area. The points are characterised by the diversified level of the sky visibility; one of them - did not have additional terrain obstacles which could limit the signal availability (except $10^{\circ}$ elevation cut-off). Firstly, for each point DOP values (N, E, V and P) were calculated within a 24-hour period (on September 24, 2013), with respect to terrain obstacles, shown in Fig. 1.

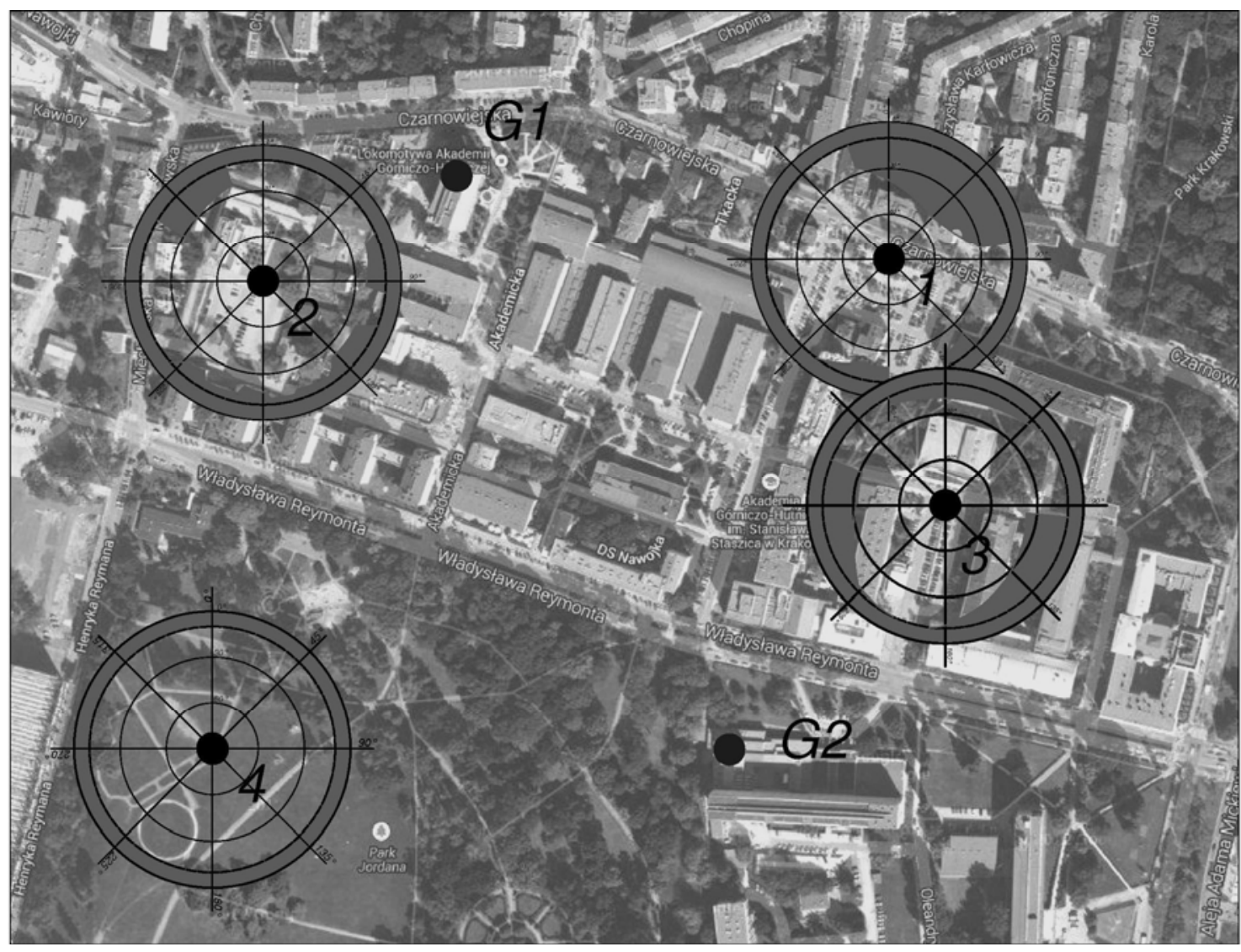

Fig. 1. Distribution of points (1-4) of the test network with visibility of the sky

Table 1 presents DOPs for each point - their maximum, minimum and averaged values for a 24-hour period. High variations of the DOP values occured within the 24-hour period. 
Table 1. Daytime values of DOPs for 4 points of the test network

\begin{tabular}{|r|r|r|r|r|r|r|r|r|r|r|r|r|}
\hline & \multicolumn{3}{|c|}{ Point 1 } & \multicolumn{3}{c|}{ Point 2 } & \multicolumn{3}{c|}{ Point 3 } & \multicolumn{3}{c|}{ Point 4 } \\
\hline & $\min$ & $\max$ & avr. & $\min$ & $\max$ & avr. & $\min$ & $\max$ & avr. & $\min$ & $\max$ & avr. \\
\hline NDOP & 0,7 & 32,9 & 1,5 & 0,7 & 2,4 & 1,5 & 0,6 & 30,2 & 1,6 & 0,5 & 2,4 & 1,0 \\
\hline EDOP & 0,5 & 32,5 & 1,4 & 0,5 & 11,7 & 1,1 & 0,5 & 24,5 & 1,1 & 0,5 & 2,8 & 0,7 \\
\hline VDOP & 1,3 & 72,1 & 3,0 & 1,1 & 136,2 & 3,1 & 1,2 & 125,6 & 3,4 & 1,1 & 4,2 & 1,8 \\
\hline PDOP & 1,8 & 85,6 & 4,4 & 1,7 & 136,8 & 4,5 & 1,6 & 131,5 & 5,0 & 1,4 & 5,4 & 2,2 \\
\hline
\end{tabular}

DOP values are useful for an initial assessment of the situation and identify the critical moments of decreased accuracy. For the purpose of displacements monitoring the main issues should be considered:

- for points 1-3 with terrain obstacles, 5-6 times during a 24-hour period the PDOP values exceeds 5 (poor conditions), sometimes reaching extreme values;

- for points 1-3 summary periods, when PDOP is not satisfied ( $>5)$ is $18 \%$ of time,

- for point 4 - with full sky visibility variations of the PDOP values between 1.4 and 5.4 occur with averaged value 2,2 ranging very good conditions; summary periods, when conditions of observations are very good (PDOP $<3$ ) is $92 \%$ of time;

- as it was expected, lacks in geometry, first of all, $\mathrm{N}$ and $\mathrm{V}$ coordinates,

In order to identify in detail the problem concerning the poor geometry, the visibility of GPS satellites in critical moments was analysed. Calculation of parameters of error ellipses allows explicit identification of poor directions. Figure 2 presents the positions of visible satellites at the moments of the highest PDOP values and directions of the long axis of error ellipses in this moment (continuous line) and at other moments of high PDOPs - above 5 (dashed line). Error ellipses are not presented due to their extremely disturbed geometry and diversified scales.

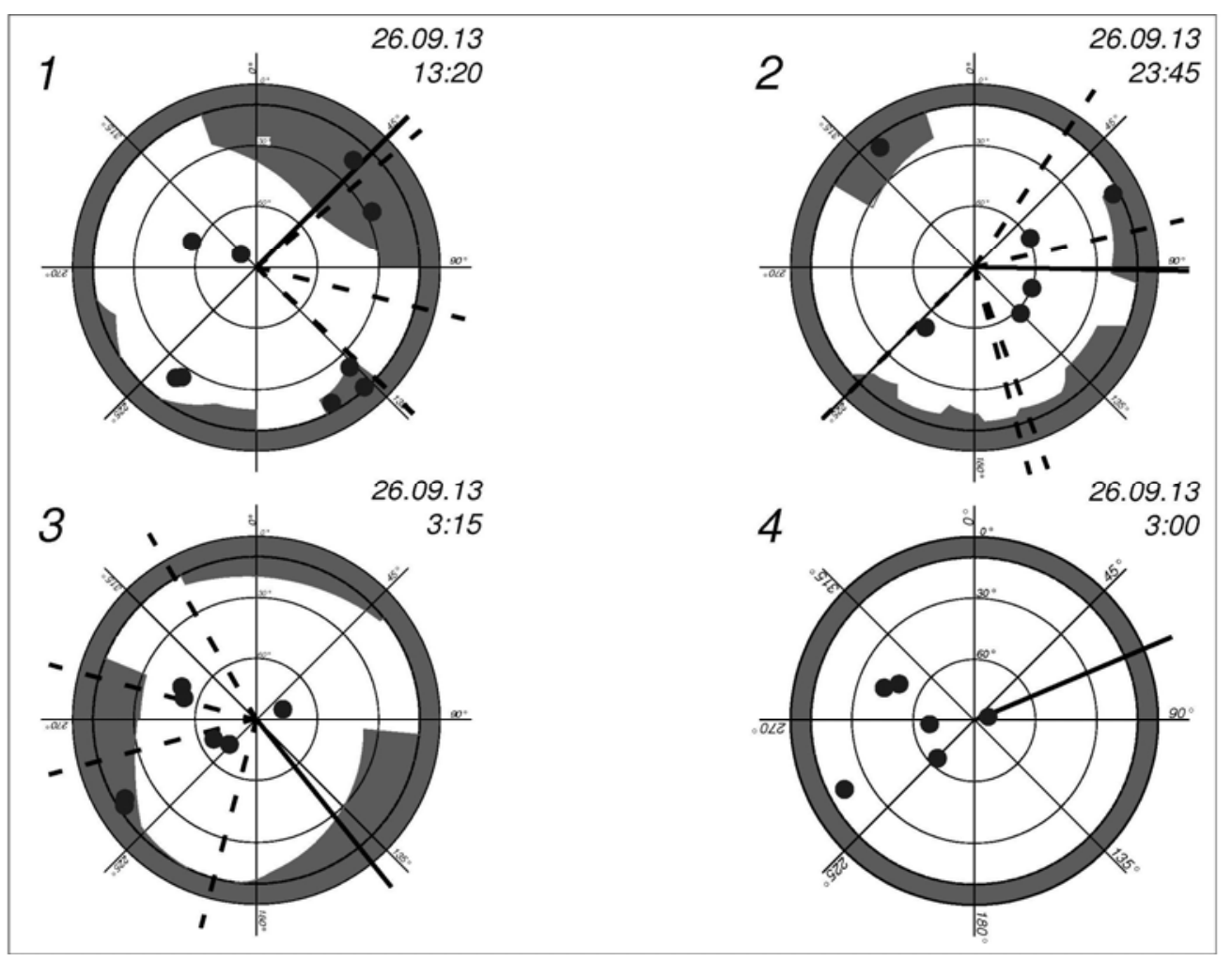

Fig. 2. Skyplots and direction of the long semi-axes of error ellipses in the critical moments 
The parameters of the maximum temporary error ellipses are specified in Table 2, when $a$, $b$ are semiaxes, and $\varphi$ - azymut of long semi-axis.

Table 2. Parameters of maximum temporary error ellipses for particular points.

\begin{tabular}{|c|c|c|c|c|}
\hline & Point 1 13:29 & Point 2 23:45 & Point 3 03:15 & Point 4 03:00 \\
\hline$a$ & 46,1 & 11,7 & 38,8 & 3,0 \\
\hline$b$ & 3,0 & 2,3 & 2,3 & 1,5 \\
\hline$\varphi$ & $44,7^{\circ}$ & $91,4^{\circ}$ & $141,0^{\circ}$ & $66,5^{\circ}$ \\
\hline
\end{tabular}

As it may be seen, the E orientation (azimuth $45^{\circ}$ do $141^{\circ}$ ) of the error ellipses is characteristic of all points, despite of ,northern observation hole”, even in point 4 (without limitation in sky visibility). In this point parameters of the ellipse is relatively low in relation to other maximum error ellipses, but the ratio between the semi-axes equal to 1:2 still exists.

For all critical moments parameters of the error ellipsoids were also calculated. Table 2 presents parameters of the maximum temporary error ellipsoids, where $A, B$ i $C$ are semiaxes, $\Phi-$ azimuth of long semi-axis projection on horizontal plane, $\mathrm{h}$ - elevation of long semi-axis in the local coordinate system.

Table 3. Parameters of maximum temporary error ellipsoids for particular points.

\begin{tabular}{|c|c|c|c|c|}
\hline & Point 1 13:29 & Point 2 23:45 & Point 3 03:15 & Point 4 03:00 \\
\hline$A$ & 85,6 & 136,7 & 131,5 & 5,0 \\
\hline$B$ & 3,3 & 2,7 & 2,5 & 1,5 \\
\hline$C$ & 0,9 & 1,2 & 1,4 & 1,1 \\
\hline$\Phi$ & $44,6^{\circ}$ & $90,2^{\circ}$ & $140,9^{\circ}$ & $66,9^{\circ}$ \\
\hline $\mathrm{h}$ & $-57,4^{\mathrm{o}}$ & $-85,1^{\circ}$ & $-72,8^{\circ}$ & $-54,8^{\circ}$ \\
\hline
\end{tabular}

There is no significant difference between the orientation of the ellipse and the ellipsoid projection in the critical moments - the ellipse/ellipsoid semi-axes are almost convergent. However, in one of the critical moments - at 7:40PM, point 2 a significant difference $\left(57^{\circ}\right)$ has occurred. That moment is presented in detail later in this paper.

Summarize, although the visibility of satellites always exceds required 4 for points $1-3$, in $18 \%$ of time the poor conditions of observations occur, what is confirmed by the values of the DOP, given in Table 1, and by the elements of the error ellipses- Table 2. In monitoring systems such break in measurements could be danger for safety of a monitored structure. Even in the point with full sky-visibility very good observation conditions occur in $92 \%$ of time, it may be unsatisfactory for the continuous precise monitoring.

\section{GROUND-BASED SOURCES OF POSITNIONING SIGNAL}

One of the solutions of issues related to the poor geometry of satellite measurements is the technology of pseudosatellites, which was the subject of wide and intensive research during the last two decade. Utilisation of ground-based, appropriately distributed transmitters of satellite signals (which are called the pseudo-satellites, pseudolites or PL) seemed to be an universal solution to the problem of lack of geometry of GPS satellites. The additional signals with different geometric conditions may highly improve a poor geometry of the GPS satellites and availability of the positioning signals (Chen, He 2006, Meng et al. 2004, Sultana et al. 2010). Positions of pseudolites may be analysed and optimised in such a way that the additional observations may improve the positioning geometry and integrity of results and, 
therefore, reduce their variations. As it was already mentioned, in the case of GPS measurements from small elevation angles are usually neglected, so placing a pseudosatellite close to the horizon may highly improve the geometry of vertical coordinate determination. On the other hand, placing the signal source in the "northern observational hole" may improve the accuracy of determination of the northern coordinate.

Although the first, promising results of research were reported, it turned out that many problems with their applications occured, so they never be in a commercial use for the surveying purposes. It was finally stated that:

- changes in the signal structure are required in order to limit the influence of many measurement errors;

- only a part of commonly used receivers may trace the signals of pseudosatellites;

- problems with time synchronisation exist;

- the problem of legality of commercial transmitting satellite signals should be considered.

Thus, if the satellite signal frequency is not appropriate for a ground-based transmitters and, additionally, the majority of the most popular receivers are not able to trace their signal, the idea to leave the assumption of transmitting the signal identical with the satellite signal was created. The terrestrial positioning technology appeared, based on the proprietary signal of the commercially available frequency, which has been selected in such a way that the influence of errors of measurements is reduced (for example Locata, Terralite). Additionally, besides transmitting the signal, the Locata-devices were coupled with the receiver, what allows synchronisation and self-calibration within the network. These devices (transceivers) may operate as supporting devices for the GPS satellite constellation, but also, they may create "a localised autonomous terrestrial replica of GNSS" (if their number at least equals to 4), which may operate independently from the satellite constellation (Rizos et al, 2011, Roberts et al. 2009). The basic advantage of that solution is the full control over the signal transmission, in particular, the positioning geometry. Supporting the satellite network, using the discussed system, may considerably widen the area and the accuracy of positioning.

Ground-based systems share characteristics with satellite measurements and offer deployment flexibility, providing a optimisation of the geometry. Observational equations in the theory of position determination have the same structure as in the case satellite observations; thus the above procedure of preliminary analyses of the accuracy using the ground-based sources, are identical.

\section{ACCURACY PREANALYSES WITH ADDITIONAL GROUND-BASED SIGNAL}

Verification of the quality of the geometry and assessment of the benefits of using error ellipsoids in the planning of ground-based transmitter was made by a number of tests and analyzes; the results are presented below.

Basing on the test area, presented in Figure 1 and directions of the lowest accuracy presented in Table 2 and 3, locations for additional, ground sources of signals (points G1 and G2) were selected on the highest buildings within the area of measurements, in such a way, that they are visible from the determined points. For points 2, 3, 4 their location is almost convergent with the weakest directions, shown in Fig. 2. The DOP values were recalculated for the same day; results are presented in Table 3. 
Table 4. 24-hour values of the DOPs for the network points with ground sources of signals.

\begin{tabular}{|r|r|r|r|r|r|r|r|r|r|r|r|r|}
\hline & \multicolumn{3}{|c|}{ Point 1 } & \multicolumn{3}{c|}{ Point 2} & \multicolumn{3}{c|}{ Point 3 } & \multicolumn{3}{c|}{ Point 4 } \\
\hline & $\min$ & $\max$ & avr. & $\min$ & $\max$ & avr. & $\min$ & $\max$ & avr. & $\min$ & $\max$ & avr. \\
\hline NDOP & 0,7 & 3,3 & 1,2 & 0,6 & 7,3 & 1,3 & 0,6 & 4,8 & 1,0 & 0,5 & 2,1 & 0,9 \\
\hline EDOP & 0,5 & 5,9 & 1,1 & 0,5 & 2,2 & 0,9 & 0,5 & 2,6 & 0,8 & 0,4 & 1,0 & 0,6 \\
\hline VDOP & 1,0 & 5,2 & 1,9 & 1,0 & 4,0 & 1,8 & 1,0 & 3,2 & 1,5 & 0,9 & 2,6 & 1,4 \\
\hline PDOP & 1,4 & 8,1 & 2,6 & 1,5 & 8,1 & 2,4 & 1,3 & 5,6 & 2,0 & 1,3 & 2,9 & 1,8 \\
\hline
\end{tabular}

The analysis of Tables 3 and 1 leads to the following conclusions:

- the considerable decrease of the maximum DOP values may be noticed; especially for the points where additional signal location was compatible with the weakest directions, but it is still unsatisfactory at point 1 and 2 ,

- the improvement of the quality of positioning geometry, is obtained within the period of 24 hours, and the better accuracy coordination between particular coordinates, in particular, on horizontal plane.

Aggregate time when observation conditions are not satisfactory (for points 1-3) decreases from $18 \%$ to $5 \%$ of $24 \mathrm{~h}$-period. For point 4 , with full sky visibility, maximum PDOP does not exceed 3 during all day, which means that observation conditions are all the time very good. The parameters of error ellipses were also recalculated for the moments of the weakest satellite constellation, with consideration of the additional positioning signal.

Table 5. List of maximum temporary error ellipses parameters for points with additional source of signal

\begin{tabular}{|c|c|c|c|c|}
\hline & $\begin{array}{c}\text { Point 1 } \\
13: 29\end{array}$ & $\begin{array}{c}\text { Point 2 } \\
8: 55\end{array}$ & $\begin{array}{c}\text { Point 3 } \\
14: 55\end{array}$ & $\begin{array}{c}\text { Point 4 } \\
19: 25\end{array}$ \\
\hline$a$ & 6,1 & 7,8 & 4,9 & 0,9 \\
\hline$b$ & 1,0 & 0,7 & 0,9 & 0,7 \\
\hline$\varphi$ & 76 & 164 & 170 & 96 \\
HDOP & 5,2 & 2,8 & 2,5 & 2,6 \\
\hline
\end{tabular}

As it may be seen, it is obtained a significant reduction in the maximum temporary ellipse parameters, together with the decrease of the ratio between semiaxes. It should be noticed that location of the additional sources resulted not only from the analysis of the weakest directions at the critical moments but first of all due to the characteristics of the terrain and assuming only 2 additional signal sources. In the case of more open area, or when more instruments are available, their locations could be planned precisely and even better results could be obtained.

Analyzing only critical moments, there was also no possibility to notice difference between the use of the error ellipse and the error ellipsoid. In order to achieve comparative results one more analysis was performed. As already mentioned, in most cases there is no significant difference between the orientation of the ellipse/ellipsoid, especially for mentioned above critical moments, but the calculation during 24-hours shows that the difference is greater than $45^{\circ}$ through $25 \%$ of the time.

Verification of the influence of the "ellipse location" and the "ellipsoid location" of the additional ground signal has been made by the calculation of the two following moments: 
1. at point 2 at 7.40PM - one of the worse cases of decreased accuracy (PDOP value 6,8), when the difference between the ellipse/ellipdoid semiaxes is $57^{\circ}$;

2. at point 4 at 11:10PM (with full sky-visibility) - the moment of the greatest difference between the ellipse/ellipdoid semiaxes $\left(115^{\circ}\right)$ during $24 \mathrm{~h}$ period, with good GPS geometry $(\mathrm{PDOP}=2,6)$

Test 1. Figure 3 presents the distribution of satellites at point 2 , at $7: 40 \mathrm{PM}$ and the error ellipse for the GPS-only measurements. The continuous line represents direction of ellipse major semi-axis (e), and the dashed line - the projection of the ellipsoid major semi-axis on horizontal plane $(\mathrm{E})$.

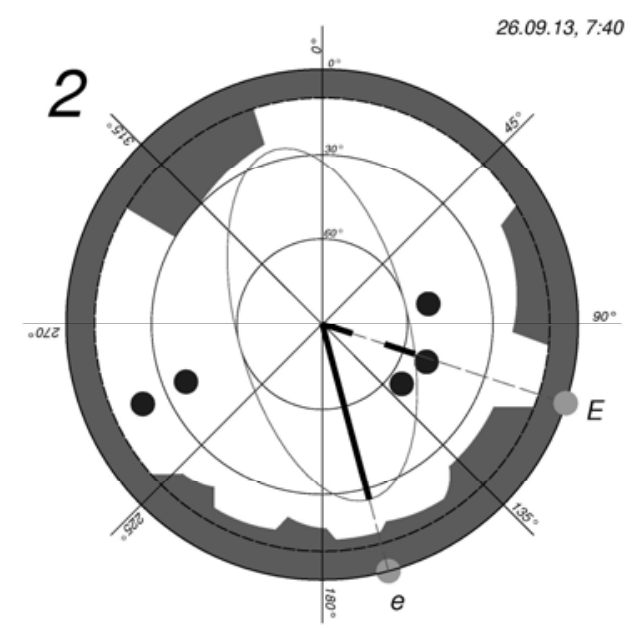

Fig. 3. Skyplot at point 2, 7:40PM with the ellipse (e) and the ellipsoid (E) semi-axes

The ground source has been located firstly in the direction indicated by the error ellipse (e), and secondly by the error ellipsoid (E). The following table summarizes the precision parameters calculated for this moment.

Table 6. Parameters of accuracy at point 2, 7:40PM (poor observational conditions)

\begin{tabular}{|c|c|c|c|c|c|c|}
\hline Parameter & \multicolumn{2}{|c|}{ GPS } & \multicolumn{2}{|c|}{$\begin{array}{l}\mathrm{GPS}+165^{\circ}(\mathrm{e}) \\
\text { 'ellipse location' }\end{array}$} & \multicolumn{2}{|c|}{$\begin{array}{l}\text { GPS }+108^{\circ}(\mathrm{E}) \\
\text { 'elipsoid location' }\end{array}$} \\
\hline DOP & $\begin{array}{l}\mathrm{N}=3,1 \\
\mathrm{E}=1,6 \\
\mathrm{~V}=5,9\end{array}$ & $\begin{array}{c}\text { PDOP } \\
6,8\end{array}$ & $\begin{array}{l}\mathrm{N}=2,6 \\
\mathrm{E}=0,7 \\
\mathrm{~V}=3,0\end{array}$ & $\begin{array}{c}\text { PDOP } \\
4,0\end{array}$ & $\begin{aligned} \mathrm{N} & =3,0 \\
\mathrm{E} & =0,7 \\
\mathrm{~V} & =1,6\end{aligned}$ & $\begin{array}{c}\text { PDOP } \\
3,4\end{array}$ \\
\hline Ellipse & $\begin{array}{l}a=3,2 \\
b=1,5\end{array}$ & $\phi=165^{\circ}$ & $\begin{array}{l}a=2,6 \\
b=0,7\end{array}$ & $\phi=5^{\circ}$ & $\begin{array}{l}a=3,0 \\
b=0,6\end{array}$ & $\phi=174^{\circ}$ \\
\hline Ellipsoid & $\begin{aligned} A & =6,1 \\
B & =3,1 \\
C & =0,7\end{aligned}$ & $\begin{array}{l}\Phi=108^{\circ} \\
\mathrm{h}=-75^{\circ}\end{array}$ & $\begin{array}{l}A=3,8 \\
B=1,0 \\
C=0,7\end{array}$ & $\begin{array}{l}\Phi=6^{\circ} \\
\mathrm{H}=-49^{\circ}\end{array}$ & $\begin{array}{l}A=3,1 \\
B=1,3 \\
C=0,6\end{array}$ & $\begin{array}{l}\Phi=174^{\circ} \\
\mathrm{H}=-16^{\circ}\end{array}$ \\
\hline
\end{tabular}

As it could be expected, additional source placed along the axis of the error ellipse introduces a significant improvement in the accuracy of the horizontal plane and slightly lower for height. However, using the ellipsoid axis improves rather the accuracy of 3-dimensional position. It could be observed a significant improvement in the accuracy of height. We obtained two times lower VDOP value than for 'ellipse direction' with a slightly lesser improvement in the horizontal plane. It should be noted that the additional source was placed in the horizon $\left(\mathrm{h}=0^{\circ}\right)$. If the environment would allow location of additional source below the measured point (below the horizon) the results may be even better. 
Test 2. The similar analysis was performed at the point with full sky visibility, when difference between the directions of ellipse/ellipsoid axes is the greatest - point 4, at 11:10PM, PDOP value is 2,6 for GPS-only mesurements.

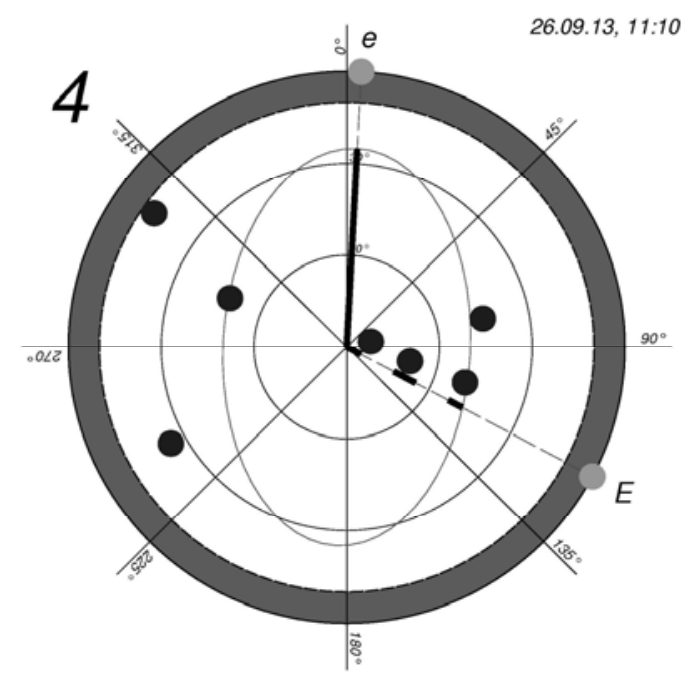

Fig. 4. Skyplot at point 4, 11:10 with ellipse (e) and ellipsoid (E) semi-axes

As in the previous case, the accuracy parameters were calculated: for GPS-only mesurements and for measurements with ground-based signal located firstly in the direction indicated by the error ellipse (e), and secondly by the error ellipsoid (E)

Table 7. Parameters of accuracy at point 4, 11:10PM (good observational conditions)

\begin{tabular}{|c|c|c|c|c|c|c|}
\hline Parameter & \multicolumn{2}{|c|}{ GPS only } & \multicolumn{2}{|c|}{$\begin{array}{l}\text { GPS }+3^{\circ}(\mathrm{e}) \\
\text { 'ellipse location' }\end{array}$} & \multicolumn{2}{|c|}{$\begin{array}{l}\text { GPS }+118^{\circ}(\mathrm{E}) \\
\text { 'elipsoid location' }\end{array}$} \\
\hline DOP & $\begin{array}{l}\mathrm{N}=1,3 \\
\mathrm{E}=0,8 \\
\mathrm{~V}=2,1\end{array}$ & $\begin{array}{l}\text { PDOP } \\
2,6\end{array}$ & $\begin{array}{l}\mathrm{N}=1,1 \\
\mathrm{E}=0,7 \\
\mathrm{~V}=1,6\end{array}$ & $\begin{array}{l}\text { PDOP } \\
2,1\end{array}$ & $\begin{array}{l}N=1,2 \\
E=0,6 \\
V=1,1\end{array}$ & $\begin{array}{l}\text { PDOP } \\
1,8\end{array}$ \\
\hline Ellipse & $\begin{array}{l}a=1,3 \\
b=0,8\end{array}$ & $\phi=3^{\circ}$ & $\begin{array}{l}a=1,2 \\
b=0,7\end{array}$ & $\phi=165^{\circ}$ & $\begin{array}{l}a=1,3 \\
b=0,5\end{array}$ & $\phi=195^{\circ}$ \\
\hline Ellipsoid & $\begin{array}{l}A=2,2 \\
B=1,3 \\
C=0,6\end{array}$ & $\begin{array}{l}\Phi=118^{\circ} \\
\mathrm{h}=-62^{\circ}\end{array}$ & $\begin{array}{l}A=1,9 \\
B=0,8 \\
C=0,6\end{array}$ & $\begin{array}{c}\Phi=158^{\circ} \\
\mathrm{H}=-58^{\circ}\end{array}$ & $\begin{array}{l}A=1,4 \\
B=1,0 \\
C=0,5\end{array}$ & $\begin{array}{l}\Phi=196^{\circ} \\
\mathrm{H}=-33^{\circ}\end{array}$ \\
\hline
\end{tabular}

The results are similar, using the error ellipsoid semi-axis it is obtained lower PDOP value, so better improvement in accuracy of 3-dimensional position. Thus, in both cases: poor and good conditions of an observation difference between using an ellipse and an ellipsoid orientation is significant for accuracy of particular coordinates. So depending on whether we determine the horizontal, vertical or spatial displacements, we should choose the proper characteristics in order to determine location of additional source. The situation, in which the difference between the directions of the ellipse and the ellipsoid are quite common (ca $25 \%$ of time) for open-sky measurements. Where the horizon obstacles appears, the distribution of satellites is much more disturbed and it could occur more frequently, so it can not be ignored while planning measurements. 


\section{CONCLUSIONS}

Within urban areas, the GPS system offers the limited continuity and accuracy of positioning, which varies in time and in space. In practice, limitations in satellite visibility may also occur, which make reliable measurements impossible. The support of the satellite network by ground-based signal transmitters increases the possibilities to apply satellite positioning in deformation monitoring by ensuring higher and more stable accuracy, and, in particular, elimination of critical moments of the decreased accuracy and ensure continuity. Insertion of additional ground-based sources of signal may solve the problem concerning the poor geometry and ensure the positioning continuity. Here, the key issue is the appropriate choice of transmitters locations. The proper location of sources of signal may significantly increase accuracy of positioning.

The proposed application of the error ellipsoids parameters instead of error ellipses or DOPs allows optimal location of additional sources and gives satisfactory results for good and poor GPS satellite geometries. The precise location of additional signals considerably increases the quality of $3 \mathrm{D}$ positioning geometry. Results of preliminary analyses of accuracy allows to obtain lower PDOP values, especially by improving vertical accuracy with a slightly lesser improvement in the horizontal plane. Application of error ellipsoid has one more advantage - by calculating orientation of the ellipse semi-axis, we do not know which direction points to the lowest accuracy $\left(\phi\right.$ or $\left.\phi+180^{\circ}\right)$. This can lead to an incorrect indication of the transmitter location. Using the error ellipsoid parameters, it is intuitively known that the semi-axis directed below the horizon pointing to the proper direction of the lowest accuracy.

The research presented in this paper does not exhaust the topic of the geometric aspects of ground augmentation of satellite networks. In the case of deformation monitoring, it would be advantageous to introduce to analyses the following elements: the complete characteristics of RDOP and relative ellipses/ellipsoids for vectors, which could give better characteristic of an estimated baseline.

\section{REFERENCES}

Aster Richard C., Borchers Brian, Thurber Clifford H. (2012) Parameter Estimation and Inverse Problems, Elsevier, 2012

Chen Y., He X., (2006) Pseudolite-augmented GPS survey technique for deformation monitoring: analysis and experimental study, 3rd IAG/12th FIG Symposium, Baden, May 22-24, 2006

Lazzarini T. oraz zespół współautorów (1977), Geodezyjne pomiary przemieszczeń budowli i ich otoczenia, $P P W K$, Warszawa 1977,

Massat P. and Rudnick K. (1990) Geometric formulas for dilution of precision calculations, Journal of the Institute of Navigation, vol. 37, No. 4, pp.379-391,

Meng X., Roberts G, Dodson A., Cosser E., Barnes J., Rizos C. (2004) Impact of GPS satellite and pseudolite geometry on structural deformation monitoring: analytical and empirical studies, Journal of Geodesy, 77,

Morales J., Khalife J. Kassas Z. (2016) GNSS Vertical Dilution of Precision Reduction using Terrestrial Signals of Opportunity, ION ITM Conference, Monterey, CA, January 25-28,

Ning F., Kao S., Chang Ch. \& Meng X., (2007) A Simulation of the Effect of GPS Pseudolite Observations on the Obstructed Sky View, Survey Review, Volume 39, Issue 303, 2007 
Rizos C., Lilly B, Robertson C., Gambale N. (2011) Open Cut Mine Machinery Automation: Going Beyond GNSS With Locata. ION GNSS 2011 Portland, Oregon, September 19-23.

Roberts G.W., Bonenberg L.K., Hancock C.M. (2009) Integrating Locatalites And GNSS For Engineering Works, 7th FIG Regional Conference, Hanoi, Vietnam, October 2009.

Rzepecka Z., Wasilewski A., Cellmer S. (2005) Integration of GPS and pseudolites: effect on the positioning accuracy, Technical Sciences, No 8, Y. 2005,

Sharp I., Yu K., Guo Y., (2009) GDOP analysis for positioning system design, IEEE Transactions on Vehicular Technology, vol. 58, no.7, pp. 3371-3382,

Sultana Q., Sunehra D., Srinivas V. and Sarma A., (2010) Effects of Pseudolite Positioning on DOP in LAAS, Positioning, Vol. 1 No. 1, 2010, pp. 18-26.

Received: 2014-01-07,

Reviewed: 2016-04-15, by J. Rapiński,

Accepted: 2016-05-10, 